\title{
Chinese in a Pandemic: TikTok as a Window Into Chinese People's Lives During COVID-19
}

\author{
Siwen $\mathrm{Lu}^{1, \mathrm{a}}$ \\ ${ }^{1}$ School of philosophy and social development, Shandong University, Shandong, China \\ ${ }^{a}$ E-mail: 516779054@qq.com
}

\begin{abstract}
TikTok, a popular short video app in China, played a significant role during the COVID-19 pandemic. Offering a window into people's daily lives against this traumatic background, TikTok was used as a research site in this study to figure out how Chinese people manifested their experiences and feelings. This study used the hashtag as a methodological tool for data selection and analyzed the videos through a thematic analysis approach. Findings indicate that Chinese people tended to express their emotional needs in TikTok videos and took a positive attitude to handle the negative feelings during the quarantine. Besides, all the videos are prone to manifest a feeling of patriotism. The study stresses the humoristic features of TikTok and the characteristics of the videos crafted according to the ethos and norms of the platform.
\end{abstract}

Keywords: TikTok, 19-COVID, social media, thematic analysis.

\section{INTRODUCTION}

TikTok (Douyin), a popular short video app in China, has witnessed drastic changes in Chinese people's everyday life since the COVID-19 pandemic broke out in January 2020. Released in 2016 by the Chinese tech giant, ByteDance, it is one of the most successful social media platforms in China. Featuring 15-to-60-second-long videos, largely constituted of user-generated content, videos on TikTok are usually embarrassing and hilarious that make people laugh. It becomes a new norm for today's Chinese people to seek entertainment and share their lives on TikTok. What's more, the special structure of TikTok ensures that what a user is less dependent on who they follow, thus regularly exposing users to new content. This structure helps grassroots video creators to be seen by more people as long as they append relevant hashtags to their videos. With its innovation and unique design, TikTok has transformed the way people interact with each other and consume digital content.

Especially, TikTok plays a significant role in people's lives in the midst of COVID-19 quarantines. With over 400 million daily active users by March 2020, up 60\% the same time last year, TikTok has experienced a meteoric rise during the pandemic. Providing access for people from all walks of life to share their own experiences, TikTok functions as a window into the life of people from different social statuses as well as helps to facilitate health communication. This study examines the special role TikTok plays during the COVID-19 pandemic, especially using it as a window to look into how Chinese people manifested their lives against the traumatic background of the COVID-19 pandemic. The ethos and norms of this platform are also paid particular attention to in a bid to see how TikTok is framing itself.

\section{LITERATURE REVIEW}

TikTok, standing out in today's swiftly moving social media app landscape, has won researchers' attention in recent years. Previous work has focused on how TikTok holds people's attention and shots to prominence among all the competitive social media platforms. Anderson[1] points out that TikTok combines the features of both Vine and Musical.ly, the former started a "stories" functionality and the latter was designed for videos of lipsynching and dancing routines. In a cinematic study, TikTok is considered to "liberate young people to play without adhering to the visual styles, narratives, and online cultures of the past"[2]. Users navigate through videos by scrolling the page, which brings about the novelty of the unknown as well as an element of uncertainty. Unable to anticipate the contents appear after swiping the page, it gives rise to chaos which is described as "nostalgic and cynical, knowing and naïve; 
manipulative, manipulated and spontaneous"[3]. Other risks such as security flaws have also raised concerns.

Similarities and distinctions between Douyin and its international twin TikTok have been underlined in some researches to investigate ByteDance's strategies to adapt its products to drastically different markets and cultures. With almost the same user interface, there do exist differences between the pages of the two apps. Its unique and involved approach to platformization in China compared with international markets contributes to its success [4]. It is especially pointed out in a study that Douyin created a separate trending section called "positive energy" for videos that promote a new form of "playful patriotism", which embodies mainstream political ideology in China [5].

During the COVID-19 pandemic, social media became a more important place to interact. Several studies have focused on social media's communication function for health promotion. Social media is considered as an important supplement to existing communication efforts [6]. The massive flow of health information health makes communication a key and necessary factor in saving lives during the COVID-19 pandemic crisis [7]. Considering its popularity and ease of adoption among community members, social media has been widely used by public health agencies to communicate health information, which is the same case with TikTok [8]. Providing verified information to help people handle anxiety and fear, official accounts on TikTok have contributed a lot during this challenging time. However, user-generated COVID-19 videos which account for the vast majority of videos uploaded on TikTok, remain unstudied so far. Therefore, this study is to figure out what are the core patterns across the videos and comments which manifest people's lives during COVID19 while looking into the special role TikTok played during this traumatic time.

\section{METHOD}

The hashtag is used as a methodological tool for data selection. TikTokers usually append hashtags deliberately for collective expression as well as to gain visibility, which facilitates the category of videos according to their themes. I first searched for the relevant keywords to identify the most popular hashtags related to COVID-19 and people's lives during the pandemic. After excluding some hashtags which contain a lot of duplication of contents that are more or less the same, I found the following ones: \#Wuhan, jiayou! (67.3 billion views), \#the frontline of fighting COVID-19 (3.25 billion views), \#fighting COVID-19 together (980 million views), \#life during the quarantine (510 million views). \#the frontline of fighting COVID-19 and \#life during the quarantine were selected as the focus of this analysis to look into the lives of frontline people and common people respectively. Furthermore, some relatively less popular hashtags were found to contain specific classes of activities, such as \#family sports meeting (240 million views), and \#concerts in communities (250 million views), which could stand for people's common behavior patterns during this special period. To ensure the timeliness and representativeness of the videos, the time range is narrowed down to the first two months in 2020.

The videos I picked were analyzed qualitatively through a thematic analysis approach, which is suitable for an investigation into people's experiences and perceptions. This method usefully summarizes key features of a large body of data, as well as highlights similarities and differences across the data set to generate unanticipated insights [9]. Videos were analyzed inductively, in the first round I coded the salient aspects in video contents and comments while making use of screenshots to capture salient phenomena. Then, considering how the data supported each theme, I summarized them into several categories. Finally, I refined my potential themes into four key themes: demand for emotional outlet and social interaction; strict compliance to the quarantine guidance; seek entertainment during the quarantine; manifestation of patriotism. The four themes are presented below with relevant examples.

\section{FINDINGS}

\subsection{Demand for Emotional Outlet and Social Interaction.}

A core pattern across videos and comments is people's desire to let out their emotions and to interact with others. During the pandemic, communities were on lockdown to slow the spread of COVID-19. Suffering from loneliness and disorientation of staying indoors all day long and fear of the intense outbreak, people took organized action and went to the balconies, opened the windows to sing and scream to cheer each other up. 


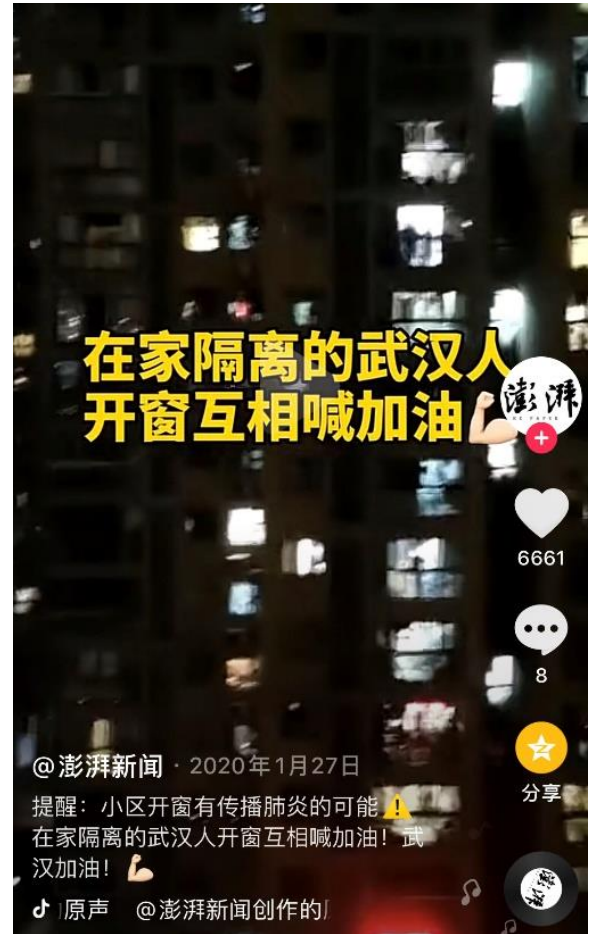

Figure 1. Videos show Wuhan citizens opening their windows to sing together or shout aloud "Stay strong, Wuhan!"

Sounds of singing and cheering rose one after another, re-echoing through the darkness. Videos recording this kind of touching scene were posted on TikTok, and they resonated with the public, the most popular one gaining $674 \mathrm{k}$ likes and $34 \mathrm{k}$ comments.

In comments, TikTokers gave high praise to the video:

Every time I see this kind of scene, my eyes are filled with tears! (@caicai)

We unite together and we can finally beat the viruses! (@yaozhiwang)

After watching videos like this, people across the country began to imitate their behaviors, videos with similar contents sprung up. Videos attached with the hashtag \#concerts in communities usually show that a man or a woman holding a microphone in the hand sang on the balcony to all the neighbors. Usually, they would receive a passionate response from the audience. People enjoying the "concert" would turn on the flashlight on their phones and wave them with the rhythm. Apart from singing and shouting, dancing was also a way of interaction. Videos recording people dance with the neighbors from the opposite building also gained much popularity on TikTok.

However, it was said by the doctors that singing in the open space would give rise to droplet transmission

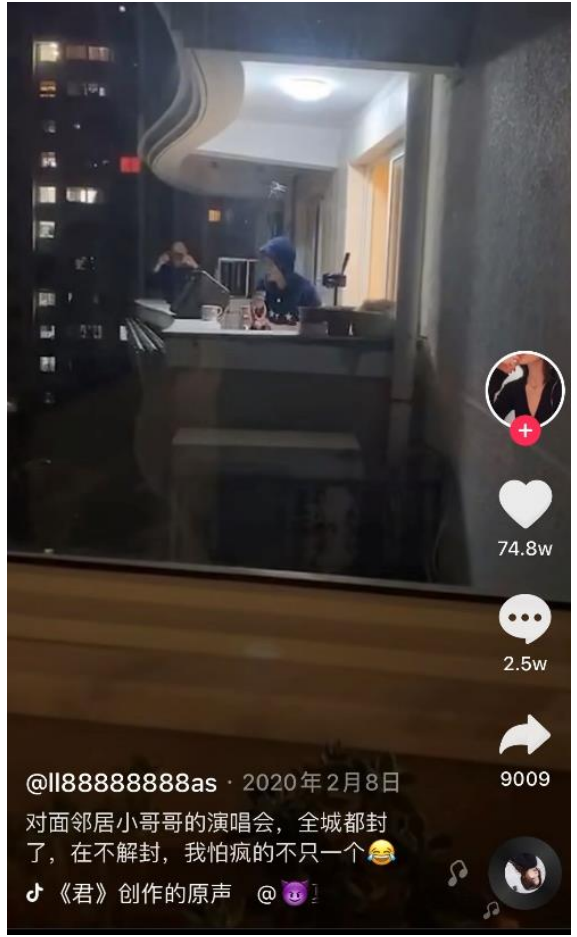

Figure 2. A video recording the "concert" of a neighbor. A man with a microphone was singing on his balcony.

between upstairs and downstairs. Official accounts made quick responses and sent warnings on TikTok to stop people from taking singing or shouting as a form of emotional outlet.

\subsection{Strict Compliance to the Quarantine Guidance.}

In fear of the spread of viruses, people strictly obeyed the government's guidance, taking all kinds of actions to stop outsiders from entering a village or community. The awareness of self-defense changed the way people commuted. In many videos, people were literally holding down the fort, using fake weapons to warn those who tried to break the rule (Figure 2). People's overreactions were common in every place during the pandemic. Even when meeting their neighbors on the stairs, people would keep the distance and then showed the health code on their phones to prove that they were healthy and had not been to high-risk areas recently (Figure 3). After seeing this hilarious scene, TikTokers did not forget to give it a thumb up in the comments:

It's right for people to have the awareness of protecting themselves. (@nannan)

Hope this pandemic ends soon and people can live a free life without all these restrictions. (@yelang) 


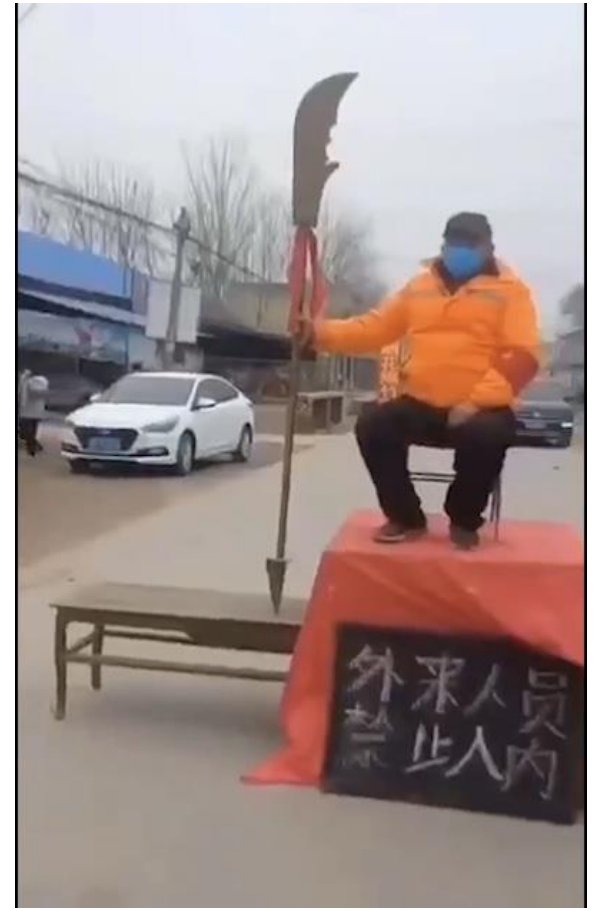

Figure 3. A video in which the security guard held a knife in his hand to stop outsiders from coming in. The blackboard says: no outsiders are allowed.

Because of COVID-19, the way Chinese people celebrated Spring Festival also changed drastically. Instead of visiting relatives, people were suggested to stay at home. A strain of videos shows that people, especially the elders who tend to be stubborn, refused to open the door for their visitors, even the one outside is their sons or daughters. It is laughable to see the visitors went back reluctantly with the gifts supposed to give to the host. In conclusion, though few of us were glad to be wary of each other all the time, these videos with very strong comedy results help make serious matters much acceptable and pleasant.

\subsection{Seek entertainment during the quarantine.}

Unable to go outside, people were finding new ways to entertain themselves indoors. There is a particularly interesting trend illustrating people making use of their daily life articles to pretend that they were taking part in the Olympic Games, including curling, weight-lifting, table tennis, swimming, and so on. "Sports equipment" was made at low cost, and the whole family was having fun taking part in these games. For instance, in the video posted by@tianyi, the father with a race number on his vest was vividly imitating an athlete trying to raise the barbell, though his barbell was actually two plastic chairs. After he successfully raised it above his head, his daughter went up and award him a gold medal. Though the game was silly, everyone in the video was having great fun.

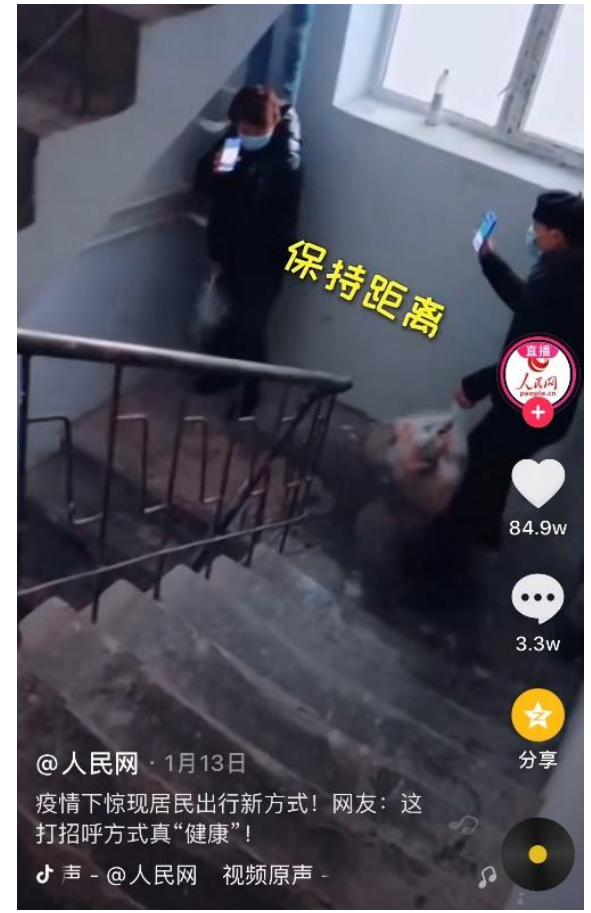

Figure 4. A video in which neighbors showed their health code to each other.

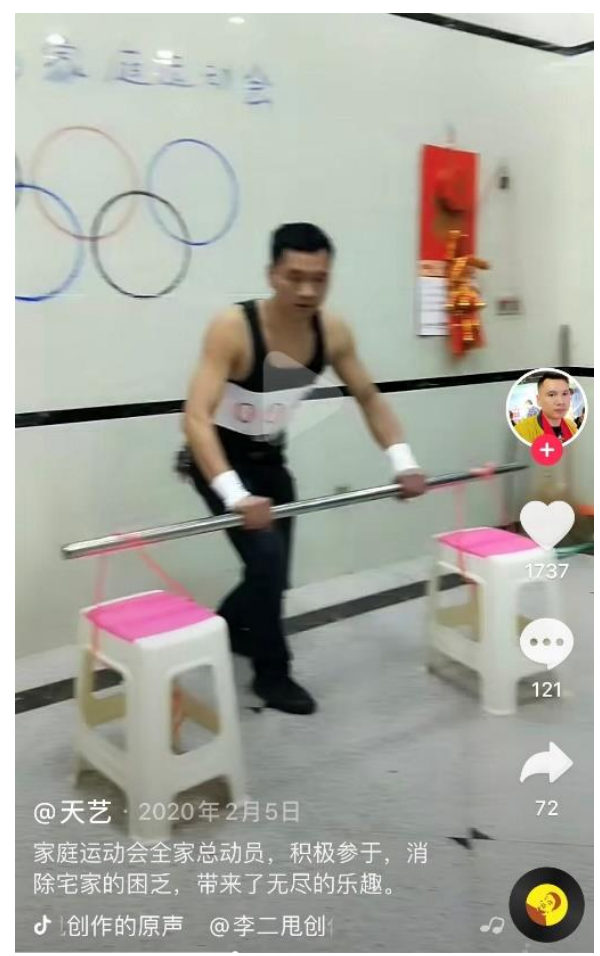

Figure 5. A videos shows that a man tried to entertain himself by imitating professional athletes at home.

Other videos are even more ridiculous. People in those videos had nothing to do but crawl on the floor like a worm, or simply set near the window to write down the number of vehicles passing by. It seemed that people were slowly driven mad during the quarantine, but they 
still had some ways to keep a good sense of humor to make the boring life fun, which is in line with the playful ethos of the platform.

\subsection{Manifestation of patriotism.}

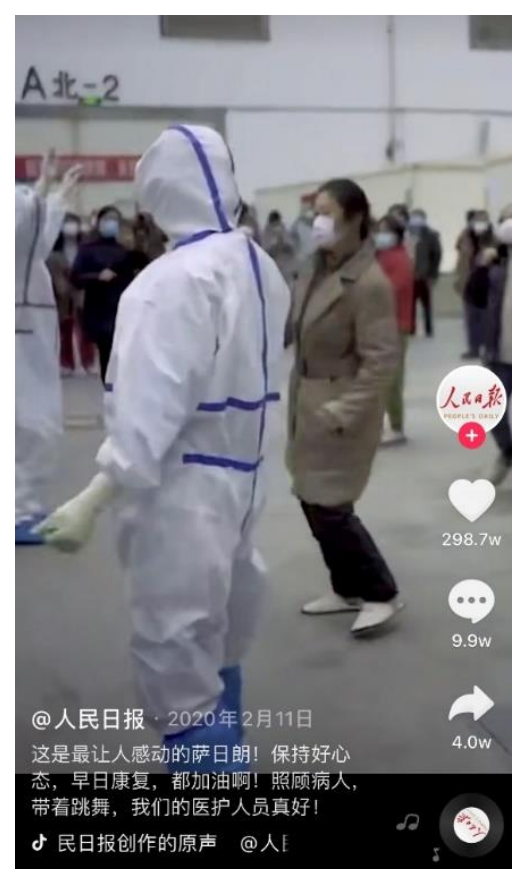

Figure 6. A video shows the nurses dancing with the patients in a makeshift hospital in Wuhan.

It has been mentioned above that when people felt emotionally connected with each other, the enthusiasm of patriotism would emerge. Videos that depicted frontline medical workers doing their best to save lives also touched people's hearts. Reporters from CCTV News interviewed some of the frontline nurses, their answers were impressive: "Like common people, we are afraid of getting infected too. But once we put on the white coats, we should try our best in our own job." In another video posted by@The People's Daily, to help patients relieve depression and improve moods, the nurses in a makeshift hospital organized the patients to dance together. People wearing masks and protective clothes danced in an atmosphere of joy, which made a huge contrast. Though obsessed with diseases and fears, they all stuck together, and hope flamed in them.

Showing people from all walks of life uniting and fighting together, the videos not only highlight the medical workers' industrious payout but also helped enhance the national sense of pride and national selfconfidence. Promoting videos embedding "positive energy" to cultivate the Chinese patriotic internet is what TikTok and other digital media platforms in China always do to comply with the regulation.

\section{CONCLUSION}

It has to be confessed that this study still has some limitations. Since all the videos were posted on TikTok, they were crafted and curated according to the ethos and norms of the platform itself, usually featuring a sense of humor or exaggerated feelings. As a result, they may not authentically reflect the experiences of Chinese people during the COVID-19 pandemic. Essentially, this research is more like a study trying to figure out how TikTok helps to repurpose reality into ironic, bite-size content which is easily spread than a study that merely looks into the real lives of Chinese people. It should be kept in mind that what this study puts particular emphasis on is how people expressed their lives during the quarantine in the context of TikTok. However, it is exactly the "filter" TikTok put on people's lives that gives special value to the days which were supposed to be filled with distressful memories.

Functioning as a significant platform for Chinese people to communicate and entertain in the context of quarantine and the COVID-19 pandemic, TikTok offers an insight into people's lives and emotions from a more optimistic perspective. Because of the special structure of TikTok, large-scale AI models determine the personalized information flows, which enables grassroots video creators to get exposure to the public without the need for advertising or an existing follower base. It makes TikTok suitable for research that looks into common people's lives during this special period.

This study contributes to an understanding of Chinese people's portrayals of their daily lives during the COVID-19 pandemic. It also sheds light on how TikTok helps to facilitate the communication between the government and the citizens during a crisis. During the quarantine, Chinese people were generally suffering from loneliness and anxiety of staying indoors, which gave rise to their demands for emotional outlet and social interaction. However, to protect themselves from getting infected, Chinese citizens overcame all kinds of difficulties to comply with the guidance from the government and medical experts, sometimes they might overreact and the results could be hilarious. Furthermore, to fight against anxiety and boredom, Chinese people found new ways to entertain themselves. The dissemination of these humoristic videos also helped the audiences to handle anxiety and fear. During the pandemic, both the common people and the government had united together and made a great contribution to fighting COVID-19, which manifested a great sense of patriotism. TikTok helped to build a bridge between people and the government, making it easier for the government to guide the public and communicate health information effectively. As we observe in this study, TikTok is not just a platform for recreation. Through the humoristic videos, the touching details in Chinese people's lives were represented to the public, which is of great value in this traumatic period. 


\section{REFERENCES}

[1] K. E. Anderson, "Getting acquainted with social networks and apps: it is time to talk about TikTok," Libr. Hi Tech News, vol. 37, no. 4, pp. 7-12, Feb. 2020, doi: 10.1108/LHTN-01-2020-0001.

[2] E. Bresnick, "Intensified Play: Cinematic study of TikTok mobile app," p. 12.

[3] E. Weil, "What Do Teenagers Learn Online Today? That Identity Is a Work in Progress," The New York Times, Nov. 13, 2019. Accessed: Apr. 09, 2021. https://www.nytimes.com/interactive/2019/11/13/m agazine/internet-teens.html

[4] D. B. V. Kaye, X. Chen, and J. Zeng, "The coevolution of two Chinese mobile short video apps: Parallel platformization of Douyin and TikTok," Mob. Media Commun., p. 205015792095212, Aug. 2020, doi: 10.1177/2050157920952120.

[5] X. Chen, D. B. Valdovinos Kaye, and J. Zeng, "PositiveEnergy Douyin: constructing 'playful patriotism' in a Chinese short-video application," Chin. J. Commun., vol. 14, no. 1, pp. 97-117, Jan.
2021, doi: 10.1080/17544750.2020.1761848.

[6] S. Ramanadhan, S. R. Mendez, M. Rao, and K. Viswanath, "Social media use by community-based organizations conducting health promotion: a content analysis," BMC Public Health, vol. 13, no. 1, p. 1129, Dec. 2013, doi: 10.1186/1471-2458-131129.

[7] A. Finset et al., "Effective health communication a key factor in fighting the COVID-19 pandemic," Patient Educ. Couns., vol. 103, no. 5, pp. 873-876, May 2020, doi: 10.1016/j.pec.2020.03.027.

[8] Y. Li, M. Guan, P. Hammond, and L. E. Berrey, "Communicating COVID-19 information on TikTok: a content analysis of TikTok videos from official accounts featured in the COVID-19 information hub," Health Educ. Res., p. cyab010, Mar. 2021, doi: 10.1093/her/cyab010.

[9] V. Braun and V. Clarke, "Using thematic analysis in psychology," Qual. Res. Psychol., vol. 3, no. 2, pp. 77-101, Jan. 2006, doi: 10.1191/1478088706qp063oa. 